\title{
Treatment of metastatic colorectal cancer: focus on panitumumab
}

\author{
This article was published in the following Dove Press journal: \\ Cancer Management and Research \\ 24 June 2015 \\ Number of times this article has been viewed
}

\author{
Rebecca Y Tay' \\ Rachel Wong ${ }^{1-3}$ \\ Eliza A Hawkes 1,3,4 \\ 'Department of Medical Oncology, \\ Eastern Health, Box Hill, VIC, \\ Australia; ${ }^{2}$ Walter and Eliza Hall \\ Institute of Medical Research, \\ Parkville, VIC, Australia; ${ }^{3}$ Faculty \\ of Medicine, Nursing and Health \\ Sciences, Monash University, \\ Melbourne, VIC, Australia; \\ ${ }^{4}$ Department of Oncology, Olivia \\ Newton John Cancer and Wellness \\ Centre, Austin Hospital, Heidelberg, \\ VIC, Australia
}

Correspondence: Eliza A Hawkes, Department of Oncology, Level 4, Olivia Newton John Cancer and Wellness Centre, Austin Hospital, Heidelberg, VIC 3084, Australia

Tel +6I 394965763

Email eliza.hawkes@onjcri.org.au
Abstract: Targeted agents are an important therapeutic option in the treatment of metastatic colorectal cancer (mCRC). Panitumumab is a recombinant, fully humanized, immunoglobulin G2 monoclonal antibody that targets the epidermal growth factor receptor (EGFR) with efficacy in $\mathrm{mCRC}$ as monotherapy and in combination with chemotherapy. Kirsten rat sarcoma (KRAS) mutation status has emerged as an important biomarker to predict response to anti-EGFR therapy. Optimal timing for panitumumab use in the mCRC treatment algorithm has not been established. This review discusses the mechanism of action, predictive biomarkers, and role of panitumumab in the treatment of mCRC.

Keywords: panitumumab, metastatic colorectal cancer, $K R A S, R A S$, EGFR, monoclonal antibody

\section{Introduction}

Colorectal cancer (CRC) is the third leading cause of cancer worldwide and accounts for $10 \%$ of all new cancer diagnoses. ${ }^{1,2}$ Twenty percent of patients will have metastatic disease at presentation and a further $30 \%$ of those diagnosed with early stage CRC will develop metastatic disease. , $^{3,4}$

The introduction of combination chemotherapy and biological agents over the past decade has led to an improvement in median overall survival (OS) from 9 months to more than 30 months for metastatic colorectal cancer (mCRC). Furthermore, surgical resection of oligometastatic disease in selected patients may lead to long-term cure. ${ }^{5,6}$ Despite these advances, 5-year OS remains at 5\%-15\%, indicating that further refinement of our current treatment strategies for mCRC, alongside the development of new therapeutics, remains a priority. ${ }^{7}$

Targeted therapy has now been incorporated into routine clinical care for mCRC. The vascular endothelial growth factor (VEGF) and epidermal growth factor (EGF) pathways are two signaling pathways central to growth and proliferation in $\mathrm{mCRC}$ for which there are well-established therapeutic targeted agents available. Up to $60 \%-70 \%$ of patients with $\mathrm{mCRC}$ receive a biological agent during their treatment course. ${ }^{8}$

Anti-VEGF agents such as bevacizumab, ziv-aflibercept, regorafenib, and ramicurimab all have efficacy in $\mathrm{mCRC}$; however, a predictive biomarker has not yet been identified. ${ }^{9-12}$ Bevacizumab, ziv-aflibercept, and regorafenib have been US Food and Drug Administration (FDA) approved for use in mCRC. Bevacizumab in combination with chemotherapy is an established standard of care in the first- and second-line settings.

Inhibition of the EGF pathway with epidermal growth factor receptor (EGFR) antibodies is also an important therapeutic strategy. Importantly, efficacy is restricted to patients 
whose tumors do not harbor Kirsten rat sarcoma (KRAS) mutations, although rat sarcoma wild-type (WT) (RAS-WT) status does not guarantee response. Two monoclonal agents, cetuximab, a immunoglobulin G1 (IgG1) mouse-human chimeric monoclonal antibody, and panitumumab, a recombinant, fully humanized, $\operatorname{IgG} 2$ monoclonal anti-EGFR antibody specifically target the EGFR pathway and have proven activity in selected mCRC patients as monotherapy and in combination with chemotherapy. ${ }^{13,14}$ Both are FDA approved for use in mCRC. This paper discusses the mechanism of action of panitumumab, current evidence for panitumumab use in CRC, and future directions in the management of mCRC.

\section{Pharmacology of panitumumab}

EGFR is a member of the human epidermal growth factor receptor (HER)-erbB family of receptor tyrosine kinases. This family also includes three other receptor tyrosine kinases; HER2/C-neu (ErbB2), HER3 (ErbB3), and HER4 (ErbB4). ${ }^{15}$

In malignant cells, activation of the EGFR initiates a downstream signaling cascade through two main axes (Figure 1). The first axis, the KRAS-RAF-mitogen-activated protein kinase (MAPK) pathway, promotes gene transcription, cell cycle progression, and proliferation. The second axis, the phosphatidylinositol 3-kinase (PI3K) pathway, results in AKT-mammalian target of rapamycin (mTOR) activation that initiates anti-apoptosis signals. These two axes remain interconnected through the p110 subunits of PI3K. Further activation of these pathways also occurs via $R A S$ protein interactions. ${ }^{15}$ The EGFR pathway plays a critical role in CRC tumorigenesis; hence, blockade of this pathway is an attractive therapeutic strategy. ${ }^{16}$

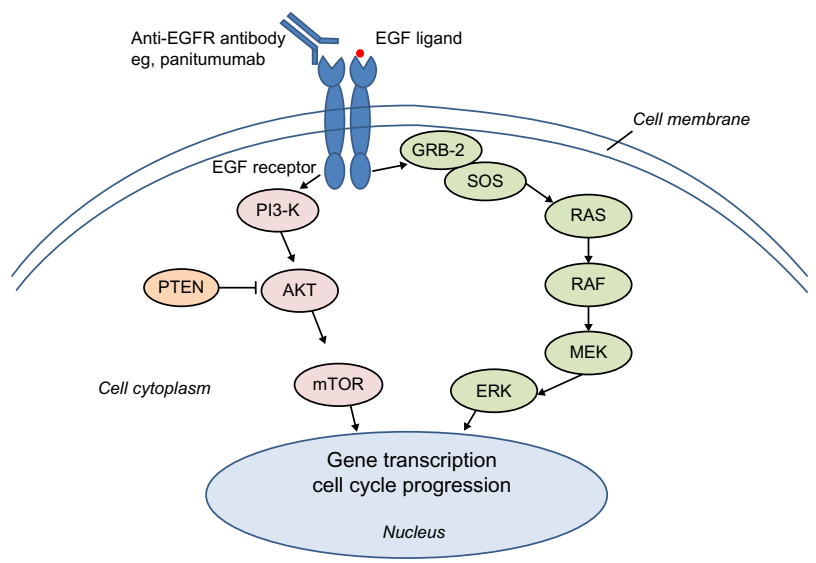

Figure I Simplified EGFR pathway.

Abbreviations: EGF, epidermal growth factor; EGFR, epidermal growth factor receptor; mTOR, mammalian target of rapamycin; PI3K, phosphatidylinositol 3-kinase.
Panitumumab binds to the extracellular domain of the EGFR, inhibiting receptor phosphorylation and subsequent activation of downstream cell signaling pathways. ${ }^{17}$ Evidence suggests panitumumab may further inhibit tumor proliferation by EGFR downregulation through receptor internalization, induction of apoptosis, autophagy, and angiogenesis inhibition..$^{17,18}$

Panitumumab is administered intravenously at a recommended dose of $6 \mathrm{mg} / \mathrm{kg}$ once every 2 weeks. An acceptable alternative dosing schedule is $9 \mathrm{mg} / \mathrm{kg}$ once every 3 weeks. ${ }^{19}$ Panitumumab exhibits nonlinear pharmacokinetics involving saturable binding to EGFR and subsequent intracellular degradation. EGFR membrane expression, gender, age, race, or renal or hepatic dysfunction does not meaningfully affect panitumumab pharmacokinetics. ${ }^{20}$ Concurrent administration of irinotecan, folinic acid/infusional 5-fluorouracil/irinotecan (FOLFIRI), or paclitaxel/carboplatin also does not alter panitumumab pharmacokinetics. ${ }^{17}$ Panitumumab is cleared via the reticuloendothelial system. ${ }^{20}$

\section{Predicting response to panitumumab}

It now well established that not all patients with $\mathrm{mCRC}$ will respond to anti-EGFR therapy. Presence in the tumor tissue of activating mutations of the $K R A S$ protein, specifically in exon 2 (codons 12 and 13) are predictive for intrinsic resistance to anti-EGFR therapy. ${ }^{21}$ Mutations of KRAS occur in 35\%-45\% of $\mathrm{mCRC}$, with proven concordance between primary and metastatic sites. ${ }^{22}$ Despite the landmark discovery of the relationship between $K R A S$ mutation status and response to anti-EGFR antibodies, the response rate in $K R A S$ wild-type (KRAS-WT) chemo-refractory patients remains in the order of $20 \%{ }^{21}$

Expanding biomarker testing beyond $K R A S$ exon 2 has offered greater insight into predicting response to anti-EGFR antibody therapy. Activating mutations in $K R A S$ exon 3 (codons 59 and 61) and exon 4 (codons 117 and 146); and neuroblastoma rat sarcoma (NRAS) exon 2 (codons 12 and 13), exon 3 (codons 59 and 61), and exon 4 (codons 117 and 146) have all been demonstrated as negative predictors of response to panitumumab. ${ }^{23} N R A S$ mutations occur in only $3 \%-5 \%$ of CRCs and are mutually exclusive of mutations in $K R A S .^{24}$ In patients who are WT for KRAS exon 2 mutations, a further $17 \%$ will exhibit a mutation in another $R A S$ exon. ${ }^{25}$

Given the predictive significance of $R A S$ mutations, determinination of $R A S$ mutation status from tumor samples is now a prerequisite prior to considering treatment with anti-EGFR 
inhibitors. ${ }^{26}$ Use of these agents in $K R A S$-mutated (KRAS-MT) tumors has been associated with worse survival outcomes. ${ }^{27}$

Other biomarkers have been less conclusive. There is a distinct lack of correlation between tumor EGFR expression measured by immunohistochemistry and response to cetuximab or panitumumab. ${ }^{28,29} B R A F$ exon V600E mutation is a known negative prognostic marker; however, while no objective response to panitumumab monotherapy has been reported in $B R A F$-mutated $\mathrm{mCRC}$, its role as a predictive marker is yet to be established..$^{30}$ EGFR gene copy number, PIK3CA mutations, or loss of PTEN expression all have been identified as potential predictive biomarkers. ${ }^{24}$ Further work is clearly warranted to improve patient selection.

\section{Evidence for clinical efficacy of panitumumab in $\mathrm{mCRC}$ Monotherapy in the chemotherapy refractory setting}

Panitumumab efficacy was initially demonstrated as monotherapy in chemotherapy-refractory mCRC. A summary of these trials is presented in Table 1. The phase III open label trial by Van Cutsem et al demonstrated a limited progression-free survival (PFS) benefit with single agent panitumumab compared to best supportive care (BSC) alone ( 8 vs 7.3 weeks, hazard ratio [HR] $0.54, P=<0.0001$ ) in an unselected KRAS population. ${ }^{31}$ No OS benefit was observed; however, any demonstrable survival benefit may have been attenuated with $76 \%$ of patients in the BSC arm crossing over to panitumumab on progression. Overall response rate (ORR) was $10 \%$, similar to that described in an earlier cetuximab study in a comparable population. ${ }^{32}$ From this study population, Amado et al further clarified the predictive role of $K R A S .{ }^{21} K R A S$ status was available in $427 / 463$ patients $(92 \%)$, with $K R A S$ exon 2 mutation detected in $43 \%$ of samples analyzed. In the $K R A S$-WT exon 2 population, panitumumab significantly improved median PFS compared to BSC (12.3 vs 7.3 weeks, HR $0.45,95 \%$ CI $0.34-0.59, P<0.0001)$. In the $K R A S$-MT population, no PFS benefit was detected (7.4 vs 7.3 weeks, HR 0.99 , 95\% CI $0.73-1.36, P$-value not specified). Response rate was $17 \%$ in the $K R A S$-WT group and $0 \%$ in $K R A S$-MT group. In a multivariate analysis, $K R A S$-WT status was a predictor for OS in both the panitumumab (HR $0.64, P=0.004$ ) and BSC arms (HR 0.68, $P=0.007$ ), the latter result suggesting a possible prognostic role; however, this has not been replicated elsewhere.

The phase III non-inferiority study ASPECCT (A Study of Panitumumab Efficacy and Safety Compared to Cetuximab in Patients with KRAS Wild-Type Metastatic Colorectal Cancer) compared panitumumab and cetuximab in 1,010 patients with chemotherapy-refractory $K R A S$-WT exon $2 \mathrm{mCRC}$ with a primary endpoint of OS. ${ }^{33}$ Panitumumab was administered at $6 \mathrm{mg} / \mathrm{kg}$ once every 2 weeks. Cetuximab was administered at an initial dose of $400 \mathrm{mg} / \mathrm{m}^{2}$, then $250 \mathrm{mg} / \mathrm{m}^{2}$ once a week thereafter. Panitumumab was proven to be non-inferior to cetuximab, with OS reported as 10.4 months and 10 months, respectively (HR 0.97, 95\% CI 0.84-1.11, $P=0.0007$ ). PFS (4.1 vs 4.4 months, HR 1.0, 95\% CI 0.88-1.14) and ORR (panitumumab at 22\% vs cetuximab at $19.8 \%$ ) were also similar. Toxicity profiles differed only slightly between the two arms. Fewer infusion reactions were reported in the panitumumab arm of any grade (3\% vs $14 \%)$ and particularly of grade $3+(<0.5 \%$ vs $2 \%)$. Higher rates of grade $3-4$ hypomagnesemia were reported with panitumumab compared to

Table I Summary of panitumumab monotherapy trials

\begin{tabular}{|c|c|c|c|c|c|c|c|}
\hline Trial & KRAS status & Treatment regimen & PFS (months) & HR (P-value) & OS (months) & HR ( $P$-value) & ORR \\
\hline \multicolumn{8}{|l|}{ Phase III } \\
\hline Van Cutsem & Unselected & BSC + Pan & 8 & $0.54(<0.000 \mathrm{I})$ & NR & 1 & $10 \%$ \\
\hline et $\mathrm{al}^{31}$ & & BSC & 7.3 & & NR & & $0 \%$ \\
\hline \multirow[t]{4}{*}{$(n=463)$} & KRAS-WT & $\mathrm{BSC}+\mathrm{Pan}$ & 12.3 & $0.45(<0.0001)$ & 8.1 & 0.99 & $17 \%$ \\
\hline & & BSC & 7.3 & & 7.6 & & $0 \%$ \\
\hline & KRAS-MT & $\mathrm{BSC}+\mathrm{Pan}$ & 7.4 & 0.99 & 4.9 & 1.02 & $0 \%$ \\
\hline & & BSC & 7.3 & & 4.4 & & $0 \%$ \\
\hline ASPECCT ${ }^{33}$ & KRAS-WT & Pan & 4.1 & I & 10.4 & $0.97(0.0007)$ & $22 \%$ \\
\hline$(n=I, 0 \mid 0)$ & exon 2 & Cetuximab & 4.4 & & 10 & & $19.8 \%$ \\
\hline \multicolumn{8}{|l|}{ Phase II } \\
\hline $\begin{array}{l}\text { Hecht et } a^{l^{42}} \\
(n=\mid 48)\end{array}$ & Unselected & Pan & I4 weeks & & 9 & & $9 \%$ \\
\hline $\begin{array}{l}\text { Muro et al }{ }^{49} \\
(n=52)\end{array}$ & Unselected & Pan & 8 weeks & & 9.3 & & $14 \%$ \\
\hline
\end{tabular}

Abbreviations: PFS, progression-free survival; HR, hazard ratio; OS, overall survival; ORR, overall response rate; BSC, best supportive care; Pan, panitumumab; NR, not reported; $n$, number of patients. 
cetuximab (7\% vs 3\%). Incidence of grade 3-4 skin toxicity was $13 \%$ and $10 \%$ in the panitumumab and cetuximab arms, respectively.

In summary, as monotherapy in chemotherapy-refractory mCRC, panitumumab has demonstrated PFS benefit in the $K R A S$-WT exon 2 population. No OS benefit was observed; however, this may in part be due to treatment cross over. ASPECCT $^{33}$ provides greater insight into use and toxicity profiles of anti-EGFR antibody monotherapy in the chemotherapy-refractory mCRC setting, given the headto-head nature of the trial design. Given the non-inferior outcomes of panitumumab compared to cetuximab, the clinician's choice of anti-EGFR agent should be considered in the context of previous treatments, cost, toxicity profile, and dosing schedules of the agents.

\section{Panitumumab in combination with chemotherapy}

First-line therapy

Chemotherapy with or without panitumumab

Panitumumab has demonstrated efficacy and acceptable toxicity when paired with both FOLFIRI and folinic acid/ infusional 5-fluorouracil/oxaliplatin (FOLFOX) chemotherapy in the first-line setting in both phase II and III trials. A summary of these trials is listed in Table 2.

The phase III PRIME study (Panitumumab Randomized trial In combination with chemotherapy for Metastatic colorectal cancer to determine Efficacy) randomized 1,183 patients to first-line FOLFOX4 with or without panitumumab (6 mg/kg every 2 weeks), with a primary endpoint of PFS. ${ }^{25} K R A S$ status was available in 1,096/1,183 (93\%) patients, with $440(40 \%)$ harboring a $K R A S$ mutation. In the
$K R A S$-WT population $(\mathrm{n}=656)$, the addition of panitumumab to FOLFOX4 resulted in a 1.4 month improvement in median PFS compared to FOLFOX4 alone (10 vs 8.6 months, HR $0.80, P=0.01)$ and an improved ORR (57\% vs $48 \%)$. No difference in ORR was detected in the KRAS-MT group.

While an initial analysis of OS in the KRAS-WT patients yielded no benefit from the addition of panitumumab, after a median follow-up of 30 months, an updated report described a significant survival advantage in KRAS-WT tumors with panitumumab + FOLFOX4 compared to FOLFOX4 alone (23.8 vs 19.4 months, HR 0.83, $P=0.03$ ). KRAS-MT patients gained no OS benefit from the addition of panitumumab with chemotherapy, with a trend toward inferior outcomes compared to chemotherapy alone (15.5 vs 19.2 months, HR $1.16, P=0.16)$. These results are consistent with the phase II OPUS study, in which the addition of cetuximab to FOLFOX chemotherapy in the KRAS-MT population also resulted in shorter median survival. ${ }^{27}$

Chemotherapy with panitumumab vs chemotherapy with bevacizumab

A first-line standard of care is bevacizumab with combination chemotherapy. ${ }^{9}$ The improved outcomes seen with first-line EGFR inhibition with chemotherapy have led to a direct comparison of these two targeted agents. Two randomized phase III studies have compared cetuximab to bevacizumab in combination with first-line chemotherapy in KRAS-WT mCRC patients, with conflicting results. The open label randomized FIRE-3 study compared FOLFIRI + cetuximab vs FOLFIRI + bevacizumab. ${ }^{5}$ In FIRE-3, no difference was seen in the primary endpoint, ORR ( $62 \%$ vs $58 \%$, odds ratio $1.18,95 \%$ CI $0.85-1.64, P=0.18$ ) or secondary endpoint,

Table 2 Summary of first-line panitumumab trials

\begin{tabular}{|c|c|c|c|c|c|c|c|}
\hline Trial & KRAS status & Treatment regimen & PFS (months) & HR (P-value) & OS (months) & HR (P-value) & ORR \\
\hline \multicolumn{8}{|l|}{ Phase III } \\
\hline PRIME $^{25}$ & KRAS-WT & FOLFOX4 + Pan & 10 & $0.8(0.01)$ & 23.8 & $0.83(0.03)$ & $57 \%$ \\
\hline \multirow[t]{3}{*}{$(n=1,183)$} & & FOLFOX4 & 8.6 & & 19.4 & & $48 \%$ \\
\hline & KRAS-MT & FOLFOX4 + Pan & 7.4 & $1.27(0.02)$ & 15.5 & $1.17(0.014)$ & $40 \%$ \\
\hline & & FOLFOX4 & 9.2 & & 19.2 & & $41 \%$ \\
\hline \multicolumn{8}{|l|}{ Phase II } \\
\hline PEAK $^{35}$ & KRAS-WT & FOLFOX + Pan & 10.9 & $0.87(0.35)$ & 34.2 & $0.62(0.009)$ & $57.8 \%$ \\
\hline$(n=285)$ & exon 2 & FOLFOX + Bev & 10.1 & & 24.3 & & $53.5 \%$ \\
\hline Kohne et $\mathrm{al}^{50}$ & Unselected & FOLFIRI + Pan & 7.6 & & NR & & $49 \%$ \\
\hline \multirow[t]{2}{*}{$(n=154)$} & KRAS-WT & FOLFIRI + Pan & 8.9 & & NR & & $56 \%$ \\
\hline & KRAS-MT & FOLFIRI + Pan & 7.2 & & NR & & $38 \%$ \\
\hline Berlin et $\mathrm{a}^{51}$ & Unselected & FOLFIRI + Pan & 10.9 & & 22.5 & & $42 \%$ \\
\hline$(n=43)$ & Unselected & $\mathrm{IFL}+\operatorname{Pan}$ & 5.6 & & 17 & & $46 \%$ \\
\hline
\end{tabular}

Abbreviations: PFS, progression-free survival; HR, hazard ratio; OS, overall survival; ORR, overall response rate; Pan, panitumumab; FOLFOX, folinic acid/infusional 5-fluorouracil/oxaliplatin; Bev, bevacizumab; FOLFIRI, folinic acid/infusional 5-fluorouracil/irinotecan; IRL, folinic acid/bolus 5-fluorouracil/irinotecan; n, number of patients; NR, not reported. 
PFS (10 vs 10.3 months, HR 1.06, 95\% CI 0.88-1.26, $P=0.55)$. A significant $\mathrm{OS}$ benefit was seen in the cetuximab group (28.7 vs 25 months, HR 0.77, 95\% CI 0.62-0.96, $P=0.017)$. In contrast, CALBG/SWOG 80405 compared FOLFIRI or mFOLFOX6 (investigator's choice) with cetuximab or bevacizumab in the KRAS-WT population. ${ }^{34}$ In the all-RAS-WT population, no OS (32 vs 31.2 months, HR 0.9, $P=0.4$ ) or PFS (11.4 vs 11.3 months, HR $1.1, P=0.31$ ) difference was detected between the cetuximab or bevacizumab groups. Higher response rates were observed in patients receiving cetuximab $(68.6 \%$ vs $53.6 \%, P=<0.01)$.

To date, the optimal first-line targeted agent for KRAS-WT patients remains unclear. While no phase III data are available comparing first-line chemotherapy with bevacizumab vs panitumumab, phase II results from PEAK (Panitumumab Efficacy in combination with mFOLFOX6 Against bevacizumab plus mFOLFOX6 in mCRC subjects with wild-type $K R A S$ tumors) provides some insight into this question. ${ }^{35}$ Similar to FIRE-3; no significant PFS difference in the panitumumab and bevacizumab arms was detected (10.9 vs 10.1 months, HR $0.87, P=0.35)$. Secondary endpoint OS was significantly improved with panitumumab (34.2 vs 24.3 months, HR 0.62, $P=0.009$ ). A pre-specified extended$R A S$ analysis, including KRAS exons 2, 3, and 4 and $N R A S$ exons 2, 3, and 4, was undertaken. Of the $K R A S$-WT exon 2 population, $60 \%$ were extended- $R A S$-WT. In the extended$R A S$-WT cohort, a trend toward improved OS was observed with panitumumab vs bevacizumab (41.3 vs 28.9 months, HR $0.63, P=0.58)$. Higher ORR was observed with panitumumab vs bevacizumab ( $57.8 \%$ vs $53.5 \%)$. Toxicity relating to the targeted agents was consistent with previous reports, with a higher incidence of skin toxicity and hypomagnesemia in the panitumumab group, and hypertension more frequently noted in the bevacizumab arm.

\section{Second-line therapy}

The combination of irinotecan-based chemotherapy and panitumumab has been evaluated in the second-line setting. Table 3 summarizes the main trials. The unblinded phase III study by Peeters et al recruited 1,186 mCRC patients who had progressed after first-line fluoropyrimidine-based chemotherapy. Patients were randomized 1:1 to FOLFIRI \pm panitumumab. ${ }^{36}$ First-line oxaliplatin and bevacizumab had been received by $67 \%$ and $19 \%$ of the study population, respectively. KRAS mutation status was available in $91 \%$ of patients, with $K R A S$ exon 2 mutation identified in $45 \%$ of tumors tested. ${ }^{8}$ Co-primary endpoints were PFS and OS. Final analysis in the KRAS-WT population $(\mathrm{n}=597$, or $55 \%$ ) demonstrated a significantly improved median PFS with the addition of panitumumab to FOLFIRI (6.7 vs 4.9 months, HR 0.82, 95\% CI 0.69-0.97, $P=0.023)$, improved ORR ( $36 \%$ vs $10 \%, P<0.0001)$, and a non-significant trend toward improved OS in favor of the panitumumab arm (14.5 vs 12.5 months, HR 0.92, $P=0.37$ ). There was no OS difference in $K R A S$-MT tumors. Of note, $34 \%$ of the $K R A S$-WT population in the FOLFIRI arm received post-progression anti-EGFR therapy.

In the phase III PICCOLO (Panitumumab, Irinotecan and Ciclosporin in COLOrectal cancer therapy) trial, 460 $K R A S$-WT mCRC patients with prior exposure to fluoropyrimidine chemotherapy were randomized to irinotecan alone or in combination with panitumumab. ${ }^{37}$ The addition of panitumumab resulted in improved PFS (HR 0.78, 95\% CI $0.64-0.95, P=0.015)$ and higher ORR (34\% vs $12 \%)$.

Table 3 Summary of second-line panitumumab trials

\begin{tabular}{|c|c|c|c|c|c|c|c|}
\hline Trial & KRAS status & Treatment regimen & PFS (months) & HR (P-value) & OS (months) & HR (P-value) & ORR \\
\hline \multicolumn{8}{|l|}{ Phase III } \\
\hline Peeters et $\mathrm{al}^{36}$ & KRAS-WT & FOLFIRI + Pan & 6.7 & $0.82(0.023)$ & 14.5 & $0.92(0.37)$ & $36 \%$ \\
\hline$(n=I, 186)$ & & FOLFIRI & 4.9 & & 12.5 & & $10 \%$ \\
\hline $\mathrm{PICCOLO}^{37}$ & KRAS-WT & Irinotecan + Pan & NR & $0.78(0.015)$ & 10.4 & I.0I (0.9I) & $34 \%$ \\
\hline$(n=460)$ & & Irinotecan & NR & & 10.9 & & $12 \%$ \\
\hline \multicolumn{8}{|l|}{ Phase II } \\
\hline SPIRITT $^{38}$ & KRAS-WT & FOLFIRI + Pan & 7.7 & I.0I (0.97) & 18 & $1.06(0.75)$ & $32 \%$ \\
\hline$n=182$ & & FOLFIRI + Bev & 9.2 & & 21.4 & & $19 \%$ \\
\hline Cohn et $\mathrm{a}^{52}$ & KRAS-WT & FOLFIRI + Pan & 6 & KRAS-WT & 11.5 & KRAS-WT & $23 \%$ \\
\hline \multirow[t]{2}{*}{$(n=116)$} & KRAS-MT & FOLFIRI + Pan & 4.3 & vs KRAS-MT & 7.1 & vs KRAS-MT & $16 \%$ \\
\hline & & & & HR 0.8 & & HR 0.6 & \\
\hline STEPP ${ }^{41}$ & KRAS-WT & Iri/FOLFIRI + Pan & 5.5 & & 13.7 & & $16 \%$ \\
\hline$(n=87)$ & KRAS-MT & Iri/FOLFIRI + Pan & 3.3 & & 13.3 & & $8 \%$ \\
\hline
\end{tabular}

Abbreviations: PFS, progression-free survival; HR, hazard ratio; OS, overall survival; ORR, overall response rate; FOLFIRI, folinic acid/infusional 5-fluorouracil/irinotecan; Pan, panitumumab; Bev, bevacizumab; Iri, Irinotecan; n, number of patients; NR, not reported. 
No significant median OS benefit was demonstrated between the panitumumab and control arms (10.4 vs 10.9 months, HR 1.01, $P=0 \cdot 91)$.

These two large randomized phase III studies demonstrate that panitumumab improves PFS and ORR in KRAS-WT $\mathrm{mCRC}$ in the second-line therapy setting. Failure to observe improved OS may, at least in part, be due the cross over and subsequent use of anti-EGFR agents.

With demonstrated benefit of adding bevacizumab and EGFR therapies to chemotherapy in the first-line and relapsed settings, the optimal targeted therapy in the second-line setting remains an ongoing question. The phase II SPIRITT (Second-line Panitumumab IRInotecan Treatment Trial) study randomized 182 patients with $K R A S$-WT mCRC who progressed on first-line FOLFOX-bevacizumab to receive second-line FOLFIRI with either panitumumab or bevacizumab. ${ }^{38}$ Improved ORR was observed in the panitumumab group compared to bevacizumab group (32\% vs 19\%). No PFS (7.7 vs 9.2 months, HR 1.01, 95\% CI $0.68-$ $1.5, P=0.97$ ) or OS (18 vs 21.4 months, HR $1.06,95 \%$ CI $0.75-1.49, P=0.75)$ benefit was demonstrated. These phase II data suggest that after progression on first-line FOLFOXbevacizumab, panitumumab does not translate to improved survival outcomes compared to bevacizumab when combined with FOLFIRI. However, expanding biomarker testing in a phase III setting may better clarify the optimal ordering of targeted agents in the second-line setting.

\section{Dual-targeted therapy: anti-VEGF plus anti-EGFR agents}

Both the VEGF and EGFR pathways are important for CRC tumorigenesis, with virtually no cross talk. Drugs targeting these have minimal overlapping toxicities, yet individually yield benefit over chemotherapy alone, regardless of the line of treatment. Dual-target blockade of both the EGFR and VEGF pathway in combination with chemotherapy was therefore assessed in the phase IIIb PACCE (Panitumumab Advanced Colorectal Cancer Evaluation) trial. ${ }^{39}$ The study randomized $1,053 \mathrm{mCRC}$ patients to receive bevacizumab plus oxaliplatin- or irinotecan- containing chemotherapy with or without panitumumab. Chemotherapy was allocated by investigator's choice, with $78 \%$ receiving oxaliplatin-based chemotherapy. KRAS mutation status (exon location not specified) was determined in $82 \%$ of patients, with a mutation found in $40 \%$ of tumor samples. In the unselected $K R A S$ population, the addition of panitumumab resulted in reduced median PFS (10 vs 11.4 months, HR 1.27, 95\% CI 1.06-1.52) and inferior median OS (19.4 vs 24.5 months, HR 1.43, 95\%
CI 1.11-1.83) compared to the control arm. Regardless of $K R A S$ mutation status, the addition of panitumumab resulted in inferior PFS compared to control. Skin toxicity occurred in $95 \%$ of patients exposed to panitumumab. Diarrhea, infection, hypomagnesemia, dehydration, and pulmonary embolism all occurred with higher frequency in the panitumumab group. Higher rates of grade $3+$ adverse events were reported in the panitumumab cohort compared to control (mean rate $90 \%$ vs $70 \%$ ). Panitumumab-related death occurred in seven (1\%) patients. Similar ORR between KRAS-WT and $K R A S$-MT tumors (50\% vs 47\%) was observed in the group receiving oxaliplatin chemotherapy, whereas a higher ORR to panitumumab was observed in the KRAS-WT vs KRAS-MT population $(54 \%$ vs $30 \%)$ in the group receiving irinotecan chemotherapy.

Several hypotheses exist that may account these poorer survival outcomes with dual-pathway inhibition. It has been proposed that the therapeutic effects of bevacizumab or chemotherapy may be blunted by EGFR inhibition via alteration to downstream targets or through cell cycle arrest leading to cytotoxic resistance. The similar response rate to panitumumab observed between $K R A S$-WT and KRAS-MT tumors in those receiving oxaliplatin chemotherapy suggests a possible interaction between oxaliplatin and panitumumab that has not been fully explored.

Significantly higher rates of toxicity may have also contributed to dose delays, lower dose intensity, and increased mortality in the panitumumab group. As extended $R A S$ testing was not specified in this trial, the true number of patients with EGFR antibody-resistant tumors remains unknown. Results of PACCE mirror the inferior outcomes and excess toxicity demonstrated in the randomized phase III CAIRO2 study, in which the addition of cetuximab to first-line capecitabine, oxaliplatin, and bevacizumab resulted in poorer PFS (9.4 vs 10.7 months, HR $1.22,95 \%$ CI $1.04-1.43, P=0.01)$ in the unselected KRAS population. In KRAS-WT tumors, no significant PFS difference was observed between the two treatment groups. ${ }^{40}$ Based on these results, combination treatment using chemotherapy plus dual-targeted agents (anti-EGFR antibody agents and bevacizumab) cannot be recommended for $K R A S$-WT patients.

\section{Safety and tolerability of panitumumab \\ Skin toxicity}

Skin toxicity is the most common adverse effect of EGFR inhibitors. Acneiform dermatitis, erythema, pruritus, dry skin, or skin fissures have all been described. Typically, rash 
and pruritus develop within the first fortnight of treatment, followed by paronychia, desquamation, and/or infections by the fourth week of treatment. ${ }^{41}$ Rash occurs in up to $90 \%$ of patients administered panitumumab. Grade 2+ toxicity has been found to correlate with improved PFS and OS. ${ }^{42}$ Management of skin toxicity has been evaluated in the randomized phase II STEPP (Skin Toxicity Evaluation Protocol with Panitumumab) study. ${ }^{41}$ Use of a pre-emptive strategy including skin moisturizers, sunscreen, topical steroids, and doxycycline for the duration of anti-EGFR therapy was reportedly well tolerated and reduced grade $2+$ skin toxicity at 6 weeks by more than $50 \%$ compared to standard care. Patients randomized to the pre-emptive strategy reported better quality of life. ${ }^{41}$

As skin toxicity may result in dose modification and discontinuation, optimizing management of skin toxicity is paramount. Withholding the subsequent panitumumab dose is recommended by the manufacturer in the event of the first occurrence of grade 3+ dermatologic reaction, despite full pre-emptive treatment. Re-challenge of panitumumab at the original dose is recommended once the reaction is deemed less than grade 3. Dose reduction is recommended by the manufacturer upon subsequent occurrence of grade $3+$ reactions. $^{14}$

\section{Hypomagnesemia}

Hypomagnesemia occurs due to EGFR inhibition in the distal convoluted tubule that usually prevents renal magnesium wasting. Panitumumab-induced hypomagnesemia occurs in up to $28 \%-36 \%$ of patients and is associated with treatment duration. ${ }^{31,39,43}$ Most cases of hypomagnesemia can be managed by oral or intravenous magnesium replacement, and rarely should it precipitate dose modification or cessation of therapy. Cardiac arrhythmia and seizure are rare but serious clinical sequelae of inadequate magnesium replacement. Hypomagnesemia is a reversible toxicity with recovery of serum magnesium levels occurring within 4-6 weeks antiEGFR therapy cessation. ${ }^{43}$ Compared to cetuximab, higher rates of hypomagnesemia (all grades) are reported with panitumumab use (27\% vs $17 \%) .{ }^{33}$

\section{Infusion reactions}

Infusion reactions are reported with most monoclonal antibodies. Severe infusion reactions with panitumumab administration occur at a rate of $2 \% .{ }^{33}$ Use of premedication with panitumumab is not routine. Fewer infusion reactions (all grades) have been reported with panitumumab compared to cetuximab ( $3 \%$ vs $14 \%$ ), consistent with the fully humanized nature of panitumumab. ${ }^{31,33}$ Grade 1 or 2 infusion reactions should prompt reduction of the infusion rate by $50 \%$. Administration of pre-medications such as antihistamines and/or corticosteroids prior to infusions can prevent further reactions in this scenario. Recurrent or severe infusion reactions, despite maximal pre-medication, should result in cessation of the infusion and in rare cases panitumumab discontinuation.

\section{Patient-reported quality of life}

A number of phase III trials have incorporated patient quality of life reported using assessments such as the Health State Index score and Overall Health Rating. ${ }^{25}$ Thus far, the addition of panitumumab to a chemotherapy backbone does not appear to result in poorer quality of life. With regard to the most common adverse event, skin toxicity, there was no detrimental effect on quality of life in patients who experienced grade $2+$ skin toxicity compared with patients with no or milder skin toxicity. ${ }^{25}$

\section{The place of Panitumumab in therapy and future directions}

Panitumumab has therapeutic efficacy in $K R A S$-WT mCRC as a first- or second-line agent in combination with chemotherapy and as monotherapy in chemotherapy-refractory disease. Improved outcomes with single agent anti-EGFR therapies in late stage disease are convincing; however, the optimal sequencing of panitumumab, namely the benefit of introduction into earlier lines of treatment, is yet to be established.

Data from the first-line, phase II PEAK study demonstrated an OS benefit with panitumumab/FOLFOX compared to bevacizumab/FOLFOX (32.2 vs 24.3 months) in the selected KRAS-WT population. There is a trend toward an even greater OS benefit in the extended $R A S$-WT cohort (41.3 vs 28.9 months). While these results support utilizing $R A S$ mutation status to direct choice of first-line targeted agent, further phase III data are required to establish the optimal first-line targeted agent in the all-RAS-WT population. ${ }^{35}$ Dual-pathway inhibition with both panitumumab and bevacizumab in combination with chemotherapy should be avoided after phase III data demonstrated inferior survival outcomes irrespective of $K R A S$ status. ${ }^{39}$

Moving forward, extended $R A S$ testing should be mandatory for all $\mathrm{mCRC}$ patients prior to considering anti-EGFR therapy. Mutations in $K R A S$ exons 3 and 4 and $N R A S$ exons 2 and 3 predict lack of response to anti-EGFR therapy, as demonstrated in the chemotherapy-refractory ASPECCT 
population, where response rates approached $0 \%$ in mutated $R A S$ tumors. ${ }^{33}$ Despite this significant advance in identification of non-responders, work remains to further define those most likely to derive benefit.

At a preclinical level, resistance to anti-EGFR agents has been demonstrated in tumors with HER2 gene amplification, low EGFR gene copy number, MET oncogene amplification, PIK3CA mutations (exon 9 and 20), or loss of function of key tumor suppressor gene PTEN. ${ }^{24,44,45}$ Circulating tumor cell status assessed between 2 and 10 weeks after initiating anti-EGFR antibody agents has also been shown to predict treatment failure in advance compared to traditional imaging modalities. ${ }^{46}$ However, data are conflicting, and validation is lacking for these potential biomarkers; therefore, they cannot be recommended for use in routine clinical care at present.

Identifiable mutations in mCRC also lend scope to developing new targeted therapies and novel combinations with existing drugs. New combinations are currently being evaluated with established agents such as combination panitumumab, irinotecan, and everolimus (http://www.clinicaltrials. gov identifier NCT01139138). Use of MET kinase inhibitors to overcome MET oncogene amplification in patients that acquire resistance to anti-EGFR therapies is a potential new strategy warranting further evaluation. ${ }^{47}$

More efficient and sensitive approaches to identify tumor mutations are also in development. Utilizing circulating tumor DNA from peripheral blood to screen for mutations allows for real-time identification of predictive or prognostic markers using tissue (blood) that is readily available. This approach requires further validation for commercial use but remains a promising approach in improving overall management of mCRC. ${ }^{48}$

\section{Conclusion}

Panitumumab is a recombinant, fully human, IgG2 monoclonal anti-EGFR antibody with an acceptable safety profile. For many years, panitumumab has remained an important agent in the treatment paradigm for $K R A S$-WT mCRC with demonstrated efficacy in the first-, second-line and chemorefractory settings. A recent advance has been the observation that the benefit of EGFR inhibitors is restricted to the all-RAS-WT population, although response rates for single agent therapy in chemorefractory patients remain in the order of $20 \%$. The future of $\mathrm{mCRC}$ research will involve optimizing the sequence of currently proven therapies while incorporating novel agents, as well as further translational work to build upon current knowledge of prognostic and predictive biomarkers and to identify additional druggable targets.

\section{Disclosure}

The authors report no conflicts of interest in this work.

\section{References}

1. Jemal A, Siegel R, Xu J, Ward E. Cancer statistics, 2010. CA Cancer J Clin. 2010;60:277-300.

2. Jemal A, Bray F, Center MM, Ferlay J, Ward E, Forman D. Global cancer statistics. CA Cancer J Clin. 2011;61(2):69-90.

3. Schmoll H, Van Cutsem E, Stein A, et al. ESMO Consensus Guidelines for management of patients with colon and rectal cancer. A personalized approach to clinical decision making. Ann Oncol. 2012;23: 2479-2516.

4. Böckelman C, Engelmann BE, Kaprio T, Hansen TF, Glimelius B. Risk of recurrence in patients with colon cancer stage II and III: a systematic review and meta-analysis of recent literature. Acta Oncol. 2015;54: $5-16$.

5. Heinemann V, von Weikersthal LF, Decker T, et al. FOLFIRI plus cetuximab versus FOLFIRI plus bevacizumab as first-line treatment for patients with metastatic colorectal cancer (FIRE-3): a randomised, open-label, phase 3 trial. Lancet Oncol. 2014;15(10):1065-1075.

6. Simmonds P, Primrose JN, Colquitt JL, Garden OJ, Poston GJ, Rees M. Surgical resection of hepatic metastases from colorectal cancer: A systematic review of published studies. Br J Cancer. 2006; 94:982-999.

7. Brenner H, Bouvier AM, Foschi R, et al; EUROCARE Working Group. Progress in colorectal cancer survival in Europe from the late 1980s to the early 21 st century: the EUROCARE study. Int J Cancer. 2012;131: 1649-1658.

8. Peeters M, Price T, Van Laethem JL. Anti-epidermal growth factor receptor monotherapy in treatment of metastatic colorectal cancer: where are we today? Oncologist. 2009;14:29-39.

9. Hurwitz H, Fehrenbacher L, Novotny W, et al. Bevacizumab plus irinotecan, fluorouracil, and leucovorin for metastatic colorectal cancer. N Engl J Med. 2004;350:2335-2342.

10. Van Cutsem E, Tabernero J, Lakomy R, et al. Addition of aflibercept to fluorouracil, leucovorin, and irinotecan improves survival in a phase III randomized trial in patients with metastatic colorectal cancer previously treated with an oxaliplatin-based regimen. $J$ Clin Oncol. 2012;30(28):3499-3506.

11. Grothey A, Van Cutsem E, Sobrero A, et al; CORRECT Study Group. Regorafenib monotherapy for previously treated metastatic colorectal cancer (CORRECT): an international, multicentre, randomised, placebocontrolled, phase 3 trial. Lancet. 2013;381:303-312.

12. Garcia-Carbonero R, Rivera F, Maurel J, et al. An open-label phase II study evaluating the safety and efficacy of ramucirumab combined with mFOLFOX-6 as first-line therapy for metastatic colorectal cancer. Oncologist. 2014;19(4):350-351.

13. Van Cutsem E, Köhne CH, Láng I, et al. Cetuximab plus irinotecan, fluorouracil, and leucovorin as first-line treatment for metastatic colorectal cancer: updated analysis of overall survival according to tumor KRAS and BRAF mutation status. J Clin Oncol. 2011;29(15): 2011-2019.

14. Vectibix ${ }^{\circledR}$ (panitumumab) [package insert]. Thousand Oaks, CA: Amgen Inc.; 2015.

15. Scaltriti M, Baselga J. The epidermal growth factor receptor pathway: a model for targeted therapy. Clin Cancer Res. 2006;12:5268-5272.

16. Keating GM. Panitumumab: a review of its use in metastatic colorectal cancer. Drugs. 2010;70:1059-1078.

17. Yang BB, Hecht JR, Malik I, et al. Pharmacokinetics (pk) of panitumumab and irinotecan were not altered after first-line panitumumab therapy with irinotecan, 5-fluorouracil, and leucovorin (IFL) in metastatic colorectal cancer (mCRC) patients. Ann Oncol. 2004;15(Suppl 3):iii83. 
18. Giannopoulou E, Antonacopoulou A, Matsouka P, Kalofonos HP. Autophagy: novel action of panitumumab in colon cancer. Anticancer Res. 2009;29(12):5077-5082.

19. Stephenson JJ, Gregory C, Burris H, et al. An open-label clinical trial evaluating safety and pharmacokinetics of two dosing schedules of panitumumab in patients with solid tumors. Clin Colorectal Cancer. 2009;8(1):29-37.

20. Yang BB, Lum P, Chen A, et al. Pharmacokinetic and pharmacodynamic perspectives on the clinical drug development of panitumumab. Clin Pharmacokinet. 2010;49(11):729-740.

21. Amado RG, Wolf M, Peeters M, et al. Wild-type KRAS is required for panitumumab efficacy in patients with metastatic colorectal cancer. J Clin Oncol. 2008;26:1626-1634.

22. Andreyev HJN, Norman AR, Cunningham D, et al. Kirsten RAS mutations in patients with colorectal cancer: The 'RASCAL II' study. Br J Cancer. 2001;85:692-696.

23. Douillard JY, Oliner KS, Siena S, et al. Panitumumab-FOLFOX4 treatment and RAS mutations in colorectal cancer. $N$ Engl J Med. 2013;369(11):1023-1034.

24. Custodio A, Feliu J. Prognostic and predictive biomarkers for epidermal growth factor receptor-targeted therapy in colorectal cancer: beyond KRAS mutations. Crit Rev Oncol Hematol. 2013;85:45-81.

25. Douillard JY, Siena S, Cassidy J, et al. Randomized, phase III trial of panitumumab with infusional fluorouracil, leucovorin, and oxaliplatin (FOLFOX4) versus FOLFOX4 alone as first-line treatment in patients with previously untreated metastatic colorectal cancer: the PRIME study. J Clin Oncol. 2010;28:4697-4705.

26. Yalcin S, Trad D, Kader YA, et al. Personalized treatment is better than one treatment fits all in the management of patients with mCRC: a consensus statement. Future Oncol. 2014;10(16):2643-2657.

27. Bokemeyer C, Bondarenko I, Hartmann JT, et al. Efficacy according to biomarker status of cetuximab plus FOLFOX-4 as first-line treatment for metastatic colorectal cancer: the OPUS study. Ann Oncol. 2011;22(7): 1535-1546.

28. Lièvre A, Bachet JB, Le Corre D, et al. KRAS mutation status is predictive of response to cetuximab therapy in colorectal cancer. Cancer Res. 2006;66:3992-3995.

29. Saltz LB, Meropol NJ, Loehrer PJ Sr, Needle MN, Kopit J, Mayer RJ. Phase II trial of cetuximab in patients with refractory colorectal cancer that expresses the epidermal growth factor receptor. J Clin Oncol. 2004;22:1201-1208.

30. Di Nicolantonio F, Martini M, Molinari F, et al. Wild-type BRAF is required for response to panitumumab or cetuximab in metastatic colorectal cancer. J Clin Oncol. 2008;26:5705-5712.

31. Van Cutsem E, Peeters M, Siena S, et al. Open-label phase III trial of panitumumab plus best supportive care compared with best supportive care alone in patients with chemotherapy-refractory metastatic colorectal cancer. J Clin Oncol. 2007;25(13):1658-1664.

32. Jonker DJ, O'Callaghan CJ, Karapetis CS, et al. Cetuximab for the treatment of colorectal cancer. 2007;357(20):2040-2048.

33. Price TJ, Peeters M, Kim TW, et al. Panitumumab versus cetuximab in patients with chemotherapy-refractory wild-type KRAS exon 2 metastatic colorectal cancer (ASPECCT): a randomised, multicentre, open-label, non-inferiority phase 3 study. Lancet Oncol. 2014;15(6):569-579.

34. Venook AP, Niedzwiecki D, Lenz HJ, et al. CALGB/SWOG 80405: Phase III trial of irinotecan/5-FU/leucovorin (FOLFIRI) or oxaliplatin/5-FU/leucovorin (mFOLFOX6) with bevacizumab (BV) or cetuximab (CET) for patients (pts) with KRAS wild-type (wt) untreated metastatic adenocarcinoma of the colon or rectum (MCRC). In: 2014 ASCO Annual Meeting; October 30-November 1, 2014; Chicago, IL. Abstract LBA3.

35. Schwartzberg LS, Rivera E, Karthaus M, et al. PEAK: a randomized, multicenter phase II study of panitumumab plus modified fluorouracil, leucovorin, and oxaliplatin (mFOLFOX6) or bevacizumab plus mFOLFOX6 in patients with previously untreated, unresectable, wild-type KRAS exon 2 metastatic colorectal cancer. J Clin Oncol. 2014;32(21): 2240-2247.
36. Peeters M, Price T, Cervantes A, et al. Final results from a randomized phase 3 study of FOLFIRI $\{+/-\}$ panitumumab for second-line treatment of metastatic colorectal cancer. Ann Oncol. 2014;25(1):107-116.

37. Seymour MT, Brown SR, Middleton G, et al. Panitumumab and irinotecan versus irinotecan alone for patients with KRAS wild-type, fluorouracil-resistant advanced colorectal cancer (PICCOLO): a prospectively stratified randomised trial. Lancet Oncol. 2013;14(8): 749-759.

38. Hecht JR, Cohn AL, Dakhil SR, et al. SPIRITT (study 20060141): a randomized phase II study of FOLFIRI with either panitumumab (pmab) or bevacizumab (bev) as second-line treatment (tx) in patients (pts) with wild-type (WT) KRAS metastatic colorectal cancer (mCRC). In: 2013 Gastrointestinal Cancers Symposium; January 24-26, 2013; San Francisco, CA. Abstract 454.

39. Hecht J, Mitchell E, Chidiac T, et al. A randomized phase IIIB trial of chemotherapy, bevacizumab, and panitumumab compared with chemotherapy and bevacizumab alone for metastatic colorectal cancer. J Clin Oncol. 2009;27(5):672-680.

40. Tol J, Koopman M, Cats A, et al. Chemotherapy, bevacizumab, and cetuximab in metastatic colorectal cancer. NEngl J Med. 2009;360(6): 563-572.

41. Lacouture ME, Mitchell EP, Piperdi B, et al. Skin toxicity evaluation protocol with panitumumab (STEPP), a phase II, open-label, randomized trial evaluating the impact of a pre-Emptive Skin treatment regimen on skin toxicities and quality of life in patients with metastatic colorectal cancer. J Clin Oncol. 2010;28(8):1351-1357.

42. Hecht JR, Patnaik A, Berlin J, et al. Panitumumab monotherapy in patients with previously treated metastatic colorectal cancer. Cancer. 2007;110(5):980-988

43. Costa A, Tejpar S, Prenen H, Van Cutsem E. Hypomagnesaemia and targeted anti-epidermal growth factor receptor (EGFR) agents. Target Oncol. 2011;6(4):227-233.

44. Martin V, Landi L, Molinari F. HER2 gene copy number status may influence clinical efficacy to anti-EGFR monoclonal antibodies in metastatic colorectal cancer patients. Br J Cancer. 2013;108(3):668-675.

45. Samuels Y, Wang Z, Bardelli A, et al. High frequency of mutations of the PIK3CA gene in human cancers. Science. 2004;304(5670):554

46. Mussella V, Pietrantonio F, Di Buduo E, et al. Circulating tumor cells as a longitudinal biomarker in patients with advanced chemorefractory, RAS-BRAF wild-type colorectal cancer receiving cetuximab or panitumumab. Int J Cancer. Epub February 20, 2015.

47. Bardelli A, Corso S, Bertotti A, et al. Amplification of the MET receptor drives resistance to anti-EGFR therapies in colorectal cancer. Cancer Discov. 2013;3(6):658-673.

48. Diaz LA Jr, Bardelli A. Liquid biopsies: genotyping circulating tumor DNA. J Clin Oncol. 2014;32(6):579-586.

49. Muro K, Yoshino T, Doi T, et al. A phase 2 clinical trial of panitumumab monotherapy in Japanese patients with metastatic colorectal cancer. Jpn J Clin Oncol. 2009;39(5):321-326.

50. Kohne CH, Hofheinz R, Mineur L, et al. First-line panitumumab plus irinotecan/5-fluorouracil/leucovorin treatment in patients with metastatic colorectal cancer. J Cancer Res Clin Oncol. 2012;138(1):65-72.

51. Berlin J, Posey J, Tchekmedyian S, et al. Panitumumab with irinotecan/ leucovorin/5-fluorouracil for first-line treatment of metastatic colorectal cancer. Clin Colorectal Cancer. 2007;6(6):427-432.

52. Cohn A, Shumaker G, Khandelwal P, et al. An open-label, single-arm, phase 2 trial of panitumumab plus FOLFIRI as second-line therapy in patients with metastatic colorectal cancer. Clin Colorectal Cancer. 2001;10(3):171-177. 


\section{Publish your work in this journal}

Cancer Management and Research is an international, peer-reviewed open access journal focusing on cancer research and the optimal use of preventative and integrated treatment interventions to achieve improved outcomes, enhanced survival and quality of life for the cancer patient. The journal welcomes original research, clinical \& epidemiological

studies, reviews \& evaluations, guidelines, expert opinion \& commentary, case reports \& extended reports. The manuscript management system is completely online and includes a very quick and fair peerreview system, which is all easy to use. Visit http://www.dovepress.com/ testimonials.php to read real quotes from published authors.

Submit your manuscript here: http://www.dovepress.com/cancer-management-and-research-journal 